\title{
Reciprocal Acoustic Transmissions: Instrumentation for Mesoscale Monitoring of Ocean Currents
}

\author{
PETER F. WORCESTER, ROBERT C. SPINDEL, MEMBER, IEEE, AND BRUCE M. HOWE \\ (Invited Paper)
}

\begin{abstract}
By simultaneously transmitting acoustic pulses in opposite directions between two points in midocean, one can separate the effects of ocean currents on acoustic propagation from the effects of sound-speed structure. Reciprocal acoustic transmissions can therefore be used to measure ocean currents. Acoustic transceivers have been designed and built to measure the mean currents between two points separated by 300 km. The equipment functioned satisfactorily during a short test conducted during 1983. Preliminary analysis of that experiment has yielded differential travel times that appear reasonable, but more work is required to relate the differential travel times to meaningful ocean-current estimates.
\end{abstract}

\section{INTRODUCTION}

$\mathbf{T}$ HE BASIC IDEA of using reciprocal acoustic transmissions to measure current is a simple one: a sound pulse traveling with a current travels faster than one traveling against a current [1]. Small-scale acoustic current meters based upon this idea have been in existence for some years. The first experiment to extend the technique to the direct measurement of mesoscale ocean currents by using transceiver separations of several hundred kilometers was conducted by the authors during August and September of 1983. This paper describes the instrumentation that was designed and built to conduct that experiment, and gives preliminary results from it.

The application of acoustic techniques to monitoring mesoscale ocean features was originally proposed by Munk and Wunsch [2]; they named the technique ocean acoustic tomography. The procedure consists of a) measuring the fluctuations in acoustic travel time between each of a number of moored acoustic sources and receivers, and b) computing the variable field of sound speed in the intermediate ocean using the techniques of linear inverse theory. Since a number of distinct ray paths with a variety of turning depths exist for each source-receiver pair, vertical ocean structure can also be inferred. Each ray represents a different depth-weighted average of the ocean. The first three-dimensional test of the technique was successfully conducted in 1981 [3], [4]. Oneway transmissions were used to monitor a $300-$ by $300-\mathrm{km}$ area centered at $26^{\circ} \mathrm{N}, 70^{\circ} \mathrm{W}$. The resulting sound-speed field can be converted to density and used to compute the geostrophic current field (for which the pressure gradient and

Manuscript received September 14, 1984; revised December 17, 1984. This research was supported by the Office of Naval Research and the National Science Foundation. Work at Scripps Institution of Oceanography was performed under ONR Contract NO0014-80-C-0217 and NSF Grant OCE-8017575. Work at Woods Hole Oceanographic Institution was performed under ONR Contracts NOO014-82-C-0152 and NO0014-84-C-0199 and NSF Grant OCE-82-14895.

P. F. Worcester and B. M. Howe are with the Scripps Institution of Oceanography, University of California, San Diego, La Jolla, CA 92093.

R. C. Spindel is with the Department of Ocean Engineering, Woods Hole Oceanographic Institution, Woods Hole, MA 02543.
Coriolis forces approximately balance), but this procedure does not yield the barotropic (depth-independent) current component [5]. Direct measurements of mesoscale currents by reciprocal transmissions, if successful, would add greatly to the power of ocean acoustic tomography by providing both the barotropic and baroclinic (depth-dependent) current fields. Using geostrophy as a constraint, one can with reciprocal transmissions improve the estimates of both the baroclinic current field and the sound-speed field [6].

The most exciting application of large-scale reciprocal transmissions may be to the measurement of relative vorticity [7]-[9]. Acoustic transmissions in opposite directions around a closed loop give one directly the circulation or, by Stoke's theorem, the areal-average relative vorticity. This quantity is quite difficult to measure using conventional techniques.

The chief difficulty encountered in performing long-range reciprocal transmissions is the small size of the expected differential travel times, typically only a few milliseconds at $300-\mathrm{km}$ range [5]. The instrumentation described here evolved from the instrumentation used in the 1981 Ocean Acoustic Tomography Experiment [10], but the precision of the traveltime measurements has been improved by approximately one order of magnitude. To achieve this improvement the narrowband acoustic source centered at $224 \mathrm{~Hz}$ used in 1981 was replaced with an acoustic source with approximately $100-\mathrm{Hz}$ bandwidth centered at $400 \mathrm{~Hz}$.

Sections II and III describe the geometry of the experiment and the signal processing used. Two different transceiver designs were employed in the experiment. Section IV describes the transceiver designed and fabricated by Woods Hole Oceanographic Institution (WHOI). The transceiver described in Section $V$ uses the same acoustic source, but has a receiver supplied by Scripps Institution of Oceanography (SIO). The principal difference between the two transceivers is that the SIO receiver has a vertical receiving array, while the WHOI receiver uses a single receiving hydrophone. It is necessary to correct one-way transmissions for travel-time changes due to mooring motion; the acoustic transponder system used for this purpose is described in Section VI. Preliminary results from the experiment are presented in Section VII.

\section{EXPERIMENT GEOMETRY}

Two moorings were deployed $300-\mathrm{km}$ apart in the Northwest Atlantic Ocean west of Bermuda (Fig. 1). The western mooring $\left(31^{\circ} 20.8^{\prime} \mathrm{N}, 71^{\circ} 46.6^{\prime} \mathrm{W}\right)$ had both SIO and WHOI transceivers. The northern mooring $\left(32^{\circ} 41.0^{\prime} \mathrm{N}, 68^{\circ} 57.8^{\prime} \mathrm{W}\right)$ had a WHOI transceiver, but the SIO unit consisted only of a 


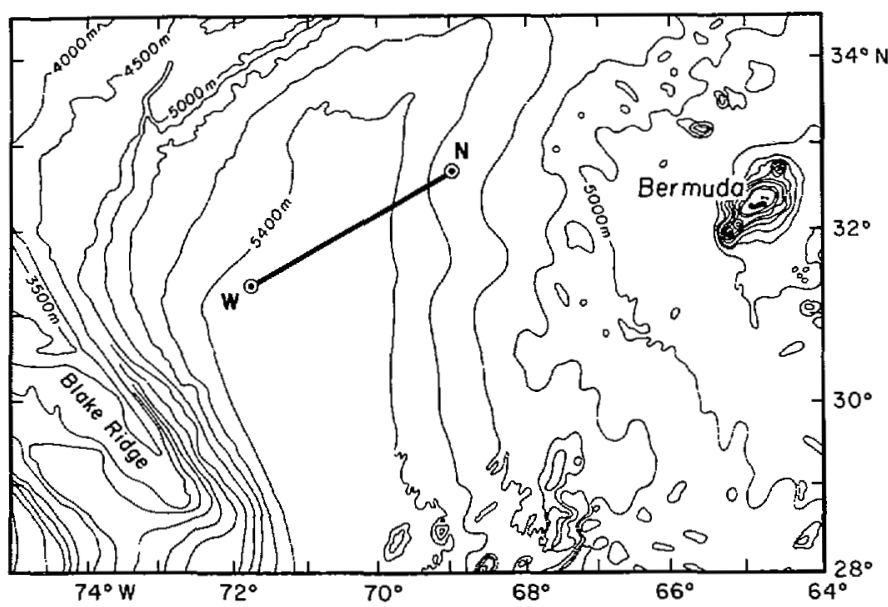

Fig. 1. Plan view of the 1983 Reciprocal Transmission Experiment. The two moorings are referred to as North and West. (The bathymetry is reproduced from Chart 5.08 of the General Bathymetric Chart of the Oceans, 5th ed., Jan. 1982.)

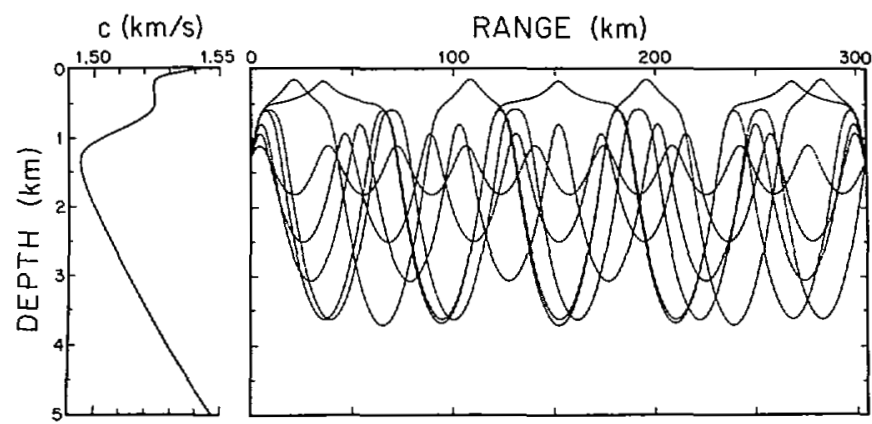

Fig. 2. Sound-speed profile (left) and a selected subset of the ray paths starting upwards from the source (right) for the 1983 Reciprocal Transmission Experiment. The source and receiver were placed near the sound channel axis, giving a wide range of upper turning-point depths.

receiver. The units were placed at approximately the depth of the sound channel axis on both moorings $(1300 \mathrm{~m})$.

The WHOI transceivers transmitted and received every two hours on the even hour (UTC). The SIO transceiver on the west mooring transmitted and received $30 \mathrm{~min}$ later, forming a reciprocal pair with the WHOI source and the SIO receiver on the northern mooring. Two sets of reciprocal transmissions therefore occurred every two hours-one on the even hour and one $30 \mathrm{~min}$ later. Twenty-two days of reciprocal data were obtained.

An average sound-speed profile appropriate for the time at which the equipment was deployed was constructed by combining data from a series of 1830 -m expendable bathythermograph (XBT) casts taken along the line joining the two moorings with historical data below $1800 \mathrm{~m}$ (Fig. 2). An historical temperature-salinity (T-S) relation was used to obtain salinity values corresponding to the XBT temperature values; sound speed was then computed using the Chen and Millero formula [11]. A selected subset of the geometric rays that start upwards at the source is displayed in Fig. 2. Rays that have a variety of upper turning depths exist for this geometry. The theoretical ray arrival structure computed for this sound-speed profile reveals the classic sofargram pattern consisting of a sequence of arrivals building toward a crescendo at the axial cutoff (Fig. 3). The WKBJ Green's

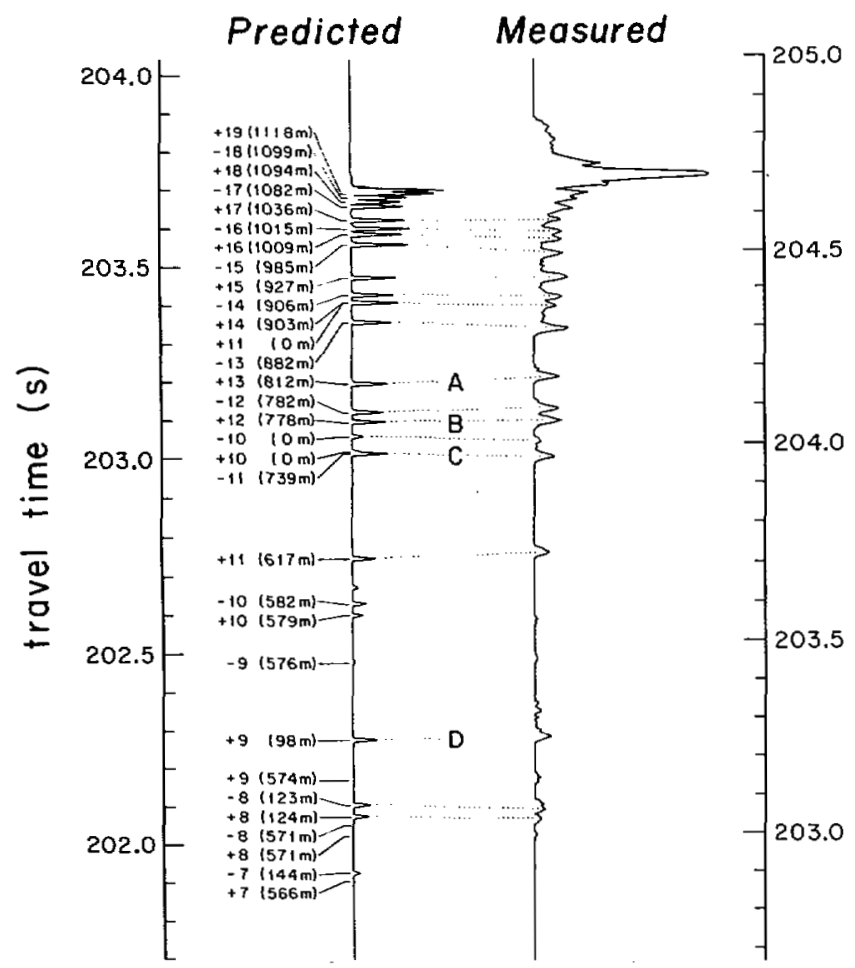

Fig. 3. Predicted (left) and measured (right) arrival amplitudes for the $\mathbf{1 9 8 3}$ Reciprocal Acoustic Transmission Experiment. Geometric ray paths are labeled with an identifier $\pm n(d \mathrm{~m})$, where $+(-)$ rays depart upwards (downwards) from the source, $n$ is the total number of upper plus lower turning points, and $d$ is the upper turning-point depth in meters. The measured arrival pattern was constructed by averaging all of the arrivals on day 217 at the SIO receiver on the North mooring after aligning them using Path $\boldsymbol{A}$. The predicted and measured travel times differ by roughly 1 $s$ because the nominal range computed from the estimated mooring locations is in error.

function technique was used to make this prediction, so both geometric and diffracted arrivals are included [12]. The curvature of the earth was properly accounted for; the range between the moorings was computed for a spheroidal earth. The earlier arrivals, which can be separated in time, form the basic data set used to compute differential travel times. It may prove possible to use a normal mode analysis to interpret the final near-axial arrivals [13].

\section{SIGNAL PROCESSING}

Pulse compression techniques are used to simultaneously achieve adequate signal-to-noise ratio and high travel-time resolution, given the available acoustic source level of $186 \mathrm{~dB}$ re $1 \mu \mathrm{Pa}$ at $1-\mathrm{m}$ rms (Table $\mathrm{I}$ ). The transmitted signal was chosen to consist of periodic repetitions of a phase-coded linear maximal shift-register sequence with the following characteristics:
a) carrier frequency $f_{0}=400 \mathrm{~Hz}$ (exactly),
b) digit length $=4$ cycles of $400 \mathrm{~Hz}=0.010 \mathrm{~s}$,
c) sequence length $L=511$ digits $=5.11 \mathrm{~s}$,
d) transmission length $=24$ sequence periods $=122.64$ $s$, and
e) modulation phase angle $\theta_{0}=87.408^{\circ}$.

The transmitted signal corresponding to a single digit in the linear maximal sequence was defined as $\sin \left(2 \pi f_{0} t \pm \theta_{0}\right)$, 
TABLE I

SIGNAL-TO-NOISE RATIO AT 300-km RANGE AND 400-Hz CENTER FREQUENCY IN THE ATLANTIC. A SINGLE HYDROPHONE RECEIVER IS ASSUMED (ATTENUATION AND NOISE LEVELS ARE FROM [14])

\begin{tabular}{|c|c|c|}
\hline $\begin{array}{l}\text { Source level (rms) } \\
\text { Spreading loss (spherical) } \\
\text { A ttenuation }(0.0168 \mathrm{~dB} / \mathrm{km})\end{array}$ & $\begin{array}{r}186 \mathrm{~dB} \\
-109.5 \mathrm{~dB} \\
-5.1 \mathrm{~dB} \\
\end{array}$ & re $1 \mu P a$ at $1 \mathrm{~m}$ \\
\hline Received signal level & $71.4 \mathrm{~dB}$ & re $1 \mu P a$ \\
\hline $\begin{array}{l}\text { Noise (1 Hz band at Sea State } 3) \\
\text { Bandwidth }(100 \mathrm{~Hz})\end{array}$ & $\begin{array}{ll}67 & \mathrm{~dB} \\
20 & \mathrm{~dB} \\
\end{array}$ & $\begin{array}{l}\text { re } 1 \mu P a / \sqrt{H z} \\
\text { re } 1 \mathrm{~Hz}\end{array}$ \\
\hline Noise level & $87 \mathrm{~dB}$ & re $1 \mu P a$ \\
\hline $\begin{array}{l}\text { A veraging gain (22 periods) } \\
\text { Processing gain (511 digits) }\end{array}$ & $\begin{array}{r}+13.4 \mathrm{~dB} \\
+27.1 \mathrm{~dB} \\
\end{array}$ & \\
\hline SNR & $24.9 \mathrm{~dB}$ & \\
\hline
\end{tabular}

where $t=0$ is the beginning of a digit and a positive (negative) phase shift corresponds to a logical " 1 " (" 0 ') in the linear maximal sequence. The modulation angle approximates the value $\theta_{0}=\arctan (\sqrt{L})=87.467^{\circ}$ required to give the line (power) spectrum of the periodic code a smooth sinc ${ }^{2}$ envelope.

This code can be processed to yield an output waveform that has no sidelobes, i.e., no self-clutter [15]. It has the additional advantage that the processing can be easily implemented on a microprocessor using only adds.

Twenty-two of the 24 sequence periods were coherently averaged in the receivers, providing $13.4 \mathrm{~dB}$ of gain; the initial and final periods were omitted from the average to avoid end effects. Correlation processing of the 511 digits in the 22period averages to yield a single $10-\mathrm{ms}$ output pulse gave another $27.1 \mathrm{~dB}$ of gain, yielding a total processing gain of $40.5 \mathrm{~dB}$ (Table I).

The rms travel-time error in a matched filter receiver using amplitude only is

$$
\sigma_{\tau}=\left[2 \pi(\Delta f)_{\mathrm{ms}} \sqrt{2 E / N}\right]^{-1}
$$

where $(\Delta f)_{\text {rms }}$ is the rms bandwidth in hertz and $2 E / N$ is the signal-to-noise ratio. The equivalent power bandwidth of the transmitted code is that of a 0.010 -s rectangular pulse, i.e., $100 \mathrm{~Hz}$. When the received code is sharply band-limited at the first spectral zeros (by the combination of the transmitter and receiver transfer functions), the rms bandwidth is $33.5 \mathrm{~Hz}$, giving $\sigma_{\tau}=0.3 \mathrm{~ms}$ for $25-\mathrm{dB}$ signal-to-noise ratio. This is consistent with the required timing precision.

"Sharp" processing, as opposed to matched filter processing, was actually implemented [16]. The received signal was complex demodulated and sampled at a two sample-per-digit rate $(200 \mathrm{~Hz})$ in the receivers. Two interlaced sequences consisting of one sample-per-digit were then constructed, separately correlated with the transmitted linear maximal sequence, and finally recombined to give the processed arrival pattern. In the frequency domain this corresponds to applying a filter with the phase response of a matched filter, but with a flat amplitude response [17], [18]. The technique sacrifices a small amount of signal-to-noise ratio to improve signal resolution. The signal-to-noise ratio degradation is not large, however, since the transfer functions of the transmitter and receiver closely approximate the amplitude response of a matched filter.

The signal processing is performed in situ in the instruments and only information about the arrival peaks is saved on magnetic tape. This approach substantially reduces the tape storage relative to that required to store unprocessed data in the receivers.

\section{WHOI TRANSCEIVERS}

A source and receiver are integrated into a single mechanical package to form the WHOI transceivers. Both operate on a common 1-MHz time base and possess RS232C-compatible serial current-loop terminations so that they can be interconnected for testing and synchronization (SAIL loop) [19].

\section{A. Acoustic-Source Transducers}

The acoustic sources evolved from the low-frequency sources that are part of the neutrally buoyant SOFAR floats used extensively to track subsurface currents in the Atlantic Ocean [20], [21]. The transducers are resonant tubes with an effective length of $\lambda / 4$, where $\lambda$ is the acoustic wavelength at $400 \mathrm{~Hz}$. Four resonators are employed, configured as two $\lambda / 2$-long tubes, each driven by a pair of flat piezoelectric elements inserted into the tube sidewalls at the midpoints. The four piezoelectric drivers are connected in parallel. Four resonators are used to broaden the bandwidth of the source relative to that obtainable with a single resonator; a 3-dB full bandwidth of approximately $100 \mathrm{~Hz}$ at a center frequency of $400 \mathrm{~Hz}$ is achieved. Source performance was verified in situ. by recording the transmitted signal at short range.

\section{B. Acoustic-Source Electronics}

Two CMOS 1802 microprocessors are employed in the source; one handles source scheduling and other control functions, while the second generates the transmitted signal (Fig. 4). An RS232C current-loop connection (SAIL loop) provides communication with the source processor. Source transmissions occur on a preset schedule. Provision is made for two separate schedules in the controller, of which one is the actual experiment schedule and the other typically provides for more frequent transmissions for a limited time to verify proper source functioning during and immediately after deployment. The transmitted signal is generated by a software algorithm that can produce a square wave at any frequency between 100 and $1000 \mathrm{~Hz}$ and any phase modulation between $\pm 90^{\circ}$ with $1-\mu$ s phase jitter (due to the basic clock rate). The phase-coded $400-\mathrm{Hz}$ digital signal is amplified by a constant power amplifier, filtered by a combination broad-band bandpass filter and impedance-matching network, and impressed upon the four ceramic drivers. Energy is supplied by alkaline batteries. 


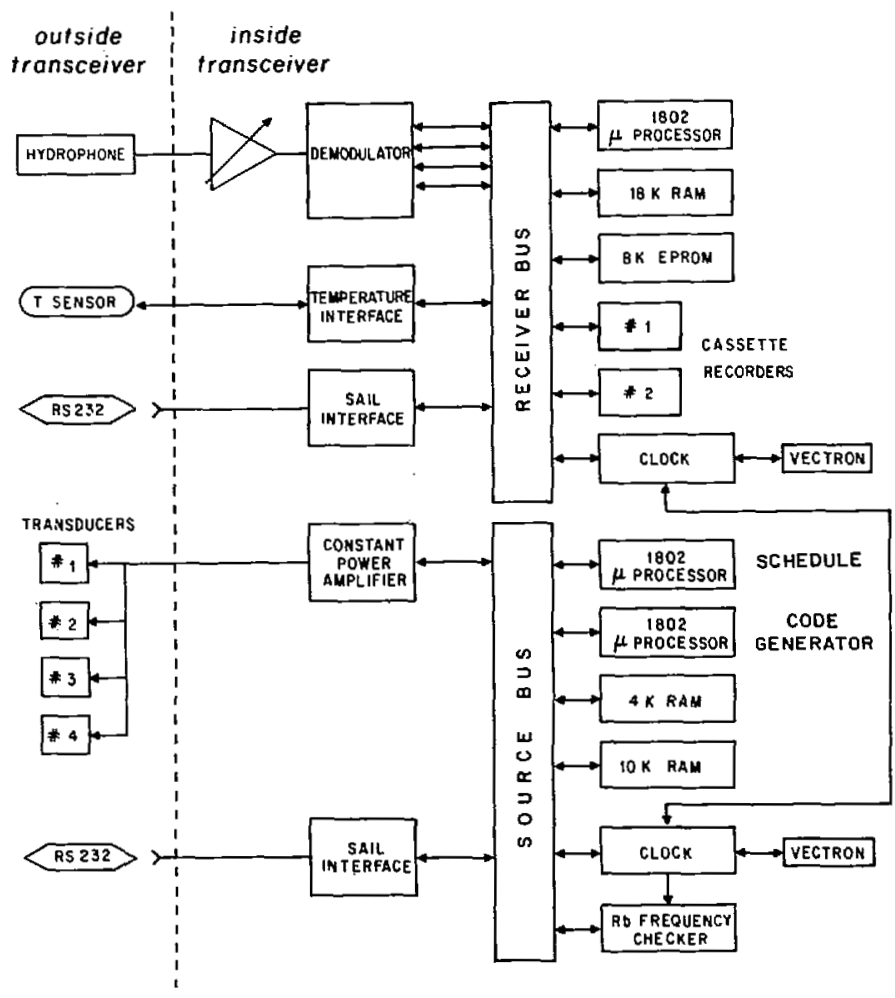

Fig. 4. Block diagram of the WHOI transceiver electronics.

The expected differential travel-time signal is only a few milliseconds. It is therefore necessary to keep time in situ with submillisecond precision for the several month duration of the experiment, requiring an oscillator accurate to better than one part in $10^{10}$. A two-oscillator system is employed in the source to achieve this precision at a reasonable level of power consumption. A low-power $(10-\mathrm{mW})$ temperature-compensated crystal oscillator (TCXO) runs continuously to drive the clock chain and other circuitry requiring a stable frequency input (including the receiver). A relatively low-power (13-W) rubidium $(\mathrm{Rb})$ atomic frequency standard, which returns to its previous frequency to within two parts in $10^{10}$ within $10 \mathrm{~min}$ after power is applied, is turned on at 6-h intervals so that the frequency difference between the Rb oscillator and the TCXO may be measured.

The frequency comparison is implemented to a precision of one part in $10^{10}$ using a phase-comparison technique. After permitting the $\mathrm{Rb}$ oscillator to warm up, the change in phase difference between the TCXO and the $\mathrm{Rb}$ oscillator is measured over a 2-minute interval and used to compute the frequency offset. The frequency offsets (Fig. 5) are stored in solid-state memory; after recovery of the instrumentation, the offsets are integrated to yield time corrections.

The magnitude of the post-experiment correction is reduced by employing feedback to adjust the TCXO frequency based on the measured frequency offset. If the measured frequency offset exceeds a preset level, the control voltage is adjusted to reduce the frequency difference. This technique increases the effective stability of the TCXO by approximately one order of magnitude, but is inadequate to permit dispensing with the post-experiment clock correction.
Functioning of the system was checked by pre- and postdeployment comparison of the source clocks with UTC provided by GOES satellite timing receivers to a precision of approximately $30 \mu \mathrm{s}$. The corrected instrument time departed from UTC by less than $2 \mathrm{~ms}$ at the conclusion of the experiment for all sources.

The SAIL-loop protocol used to communicate with the microprocessor providing scheduling and control functions is also used to set, read, and synchronize the source clock to within $10 \mu \mathrm{s}$.

\section{WHOI Receivers}

The WHOI receivers are microprocessor-controlled units that digitize, process, and record on magnetic tape acoustic data from a single hydrophone that is part of the transceiver package. The source transducers could in principle be used for reception also, but it was more convenient to use a separate hydrophone for this application. In addition, each receiver measures temperature for use in subsequent data analysis.

A single CMOS 1802 microprocessor performs both control and signal-processing functions in the receiver (Fig. 4). An RS232C current-loop connection (SAIL loop) identical to that in the source electronics is provided for communication with the processor. Both the source and receiver can then be connected in the same loop for clock synchronization purposes.

Signal acquisition is initiated by the processor at preset times computed by adding the propagation delay for the nominal range to the source transmit times. Approximately one sequence length is added to the propagation delay so that data acquisition will start near the beginning of the second period transmitted; this avoids end effects in the processing.

The incoming signal is amplified and filtered using a broad-band filter centered at $400 \mathrm{~Hz}$. The filter is designed to have little effect on the signal while reducing noise from frequencies well outside the band of interest. The filtered signal is then shifted to baseband using a square-wave complex demodulator. A novel approach has been implemented to digitize the resulting quadrature components of the signal (Fig. 6). Each component is low-pass filtered and digitized using voltage-to-frequency converters followed by counters. Each sample is the result of a 10 -ms integration, giving a frequency response closely matched to that of the transmitted signal. Two voltage-to-frequency converters are employed per component so that overlapping integrations can be simultaneously performed. A 5-ms sample interval is thus achieved, giving two samples per digit. Since there are two quadrature components and 511 digits, a single period consists of 1022 complex samples.

The processor coherently sums 22 values at each of the 1022 time positions, one from each period. The resulting average period is then "sharp" processed and the 12 largest peaks in the arrival pattern located. The arrival time and amplitude of each peak and the immediately adjacent amplitudes are stored on magnetic tape for subsequent analysis on shore. An incremental digital cassette tape recorder manufactured by Sea-Data provides $15 \mathrm{Mbit}$ of data storage, which is adequate 


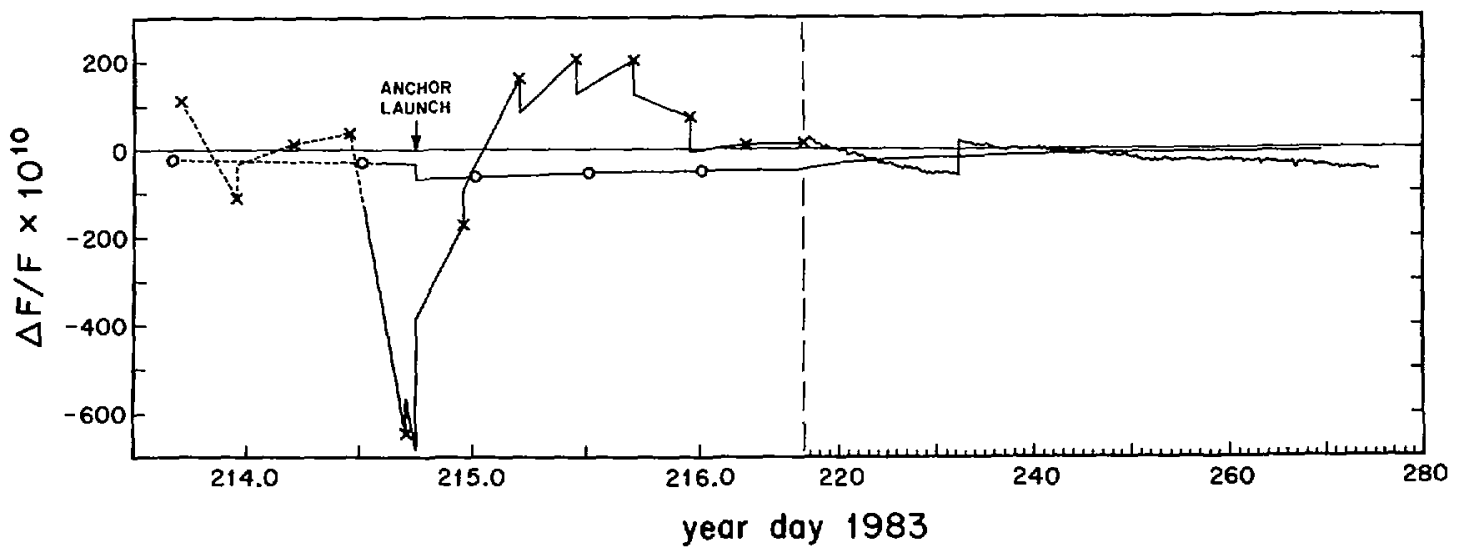

Fig. 5. Measured frequency offsets for the transceivers on the West mooring. Measured frequency offsets for the temperature-compensated crystal oscillator in the WHOI transceiver are indicated by crosses; if the measured offsets exceeded a preset value, the oscillator frequency was electronically adjusted, as indicated in the figure. Measured frequency offsets for the ovenized crystal oscillator in the SIO transceiver are marked with open circles. The frequency offsets are dashed prior to the time at which the clocks were last reset. The horizontal time scale is expanded to better display the launch transients. The interpolated curves represent a best guess of oscillator behavior during launch.

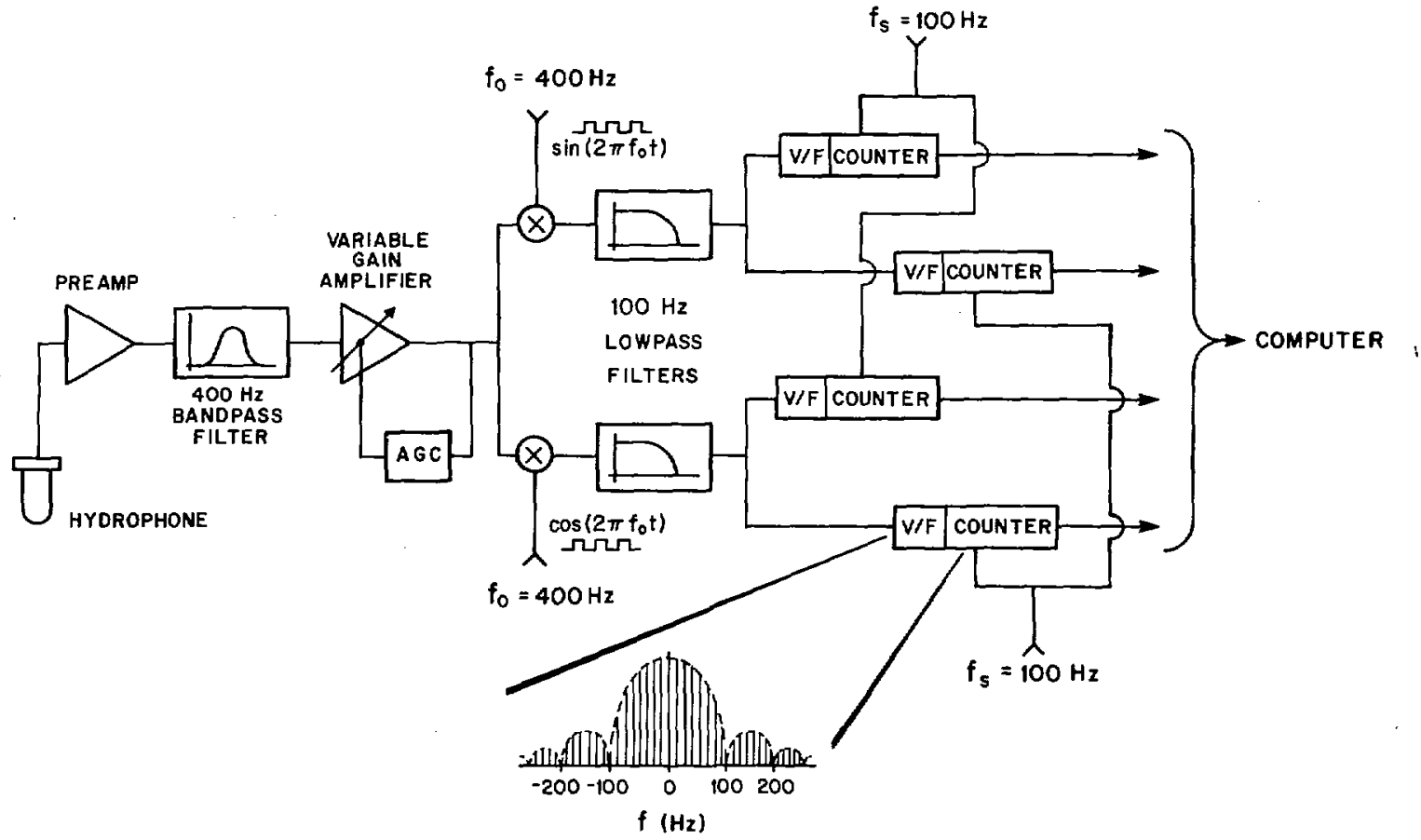

Fig. 6. Block diagram of the analog square-wave demodulator employed in the WHOI receivers to shift the received signal to baseband. The transfer function of the voltage-to-frequency converters and counters used to digitize the quadrature components of the signal is displayed.

for approximately one year. Two recorders are used in parallel for redundance. Many tape drives would have been required to store the acoustic data if it had not been processed in situ.

\section{SIO TRANSCEIVERS}

The principle functional difference between the WHOI and SIO transceivers is that the SIO receiver has a four-element vertical receiving array instead of a single receiving hydrophone. The SIO and WHOI receivers evolved separately, however, and there are therefore a number of less significant differences that are of interest.

The source and receiver are packaged separately in the SIO transceivers and connected by the electromechanical cable required for the four-element vertical hydrophone array (Fig. 7). The source is identical to that employed in the WHOI transceivers, except that it is slaved to the SIO receiver instead of being autonomous. Each transmission is triggered by a pulse from the SIO receiver and no internal timing is performed by the source. 


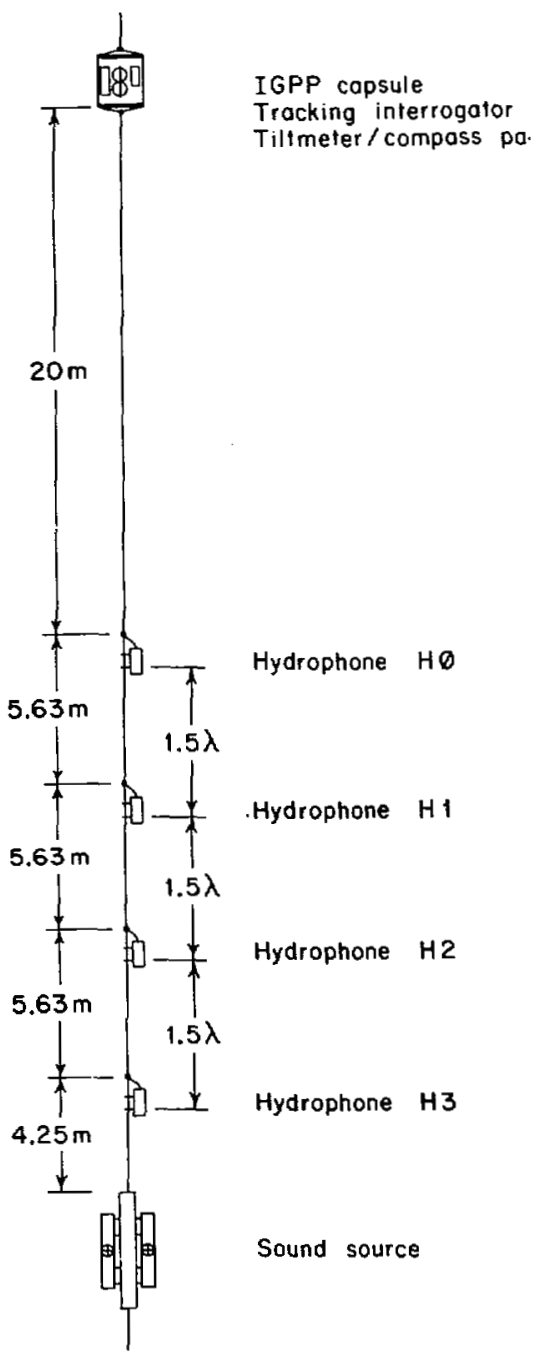

Fig. 7. Mechanical drawing of the SIO transceivers.

\section{A. SIO Receivers}

The SIO receivers are microprocessor-controlled units that digitize, process, and record on magnetic tape acoustic data from four-element vertical hydrophone arrays. Data from each hydrophone channel are recorded separately so that beams can be formed at any desired angle after the instrument is recovered. In addition, each receiver:

a) measures pressure and temperature at 15 -min intervals,

b) measures the orientation (compass) and orthogonal tilts ( $X$ and $Y$ tiltmeters) of the hydrophone array in conjunction with each acoustic reception,

c) measures the drift of the low-power crystal oscillator used in the receiver at 12-h intervals by comparing it to a $\mathbf{R b}$ frequency standard,

d) triggers the acoustic source at preset times, and

e) provides for in situ high-frequency acoustic communications with a surface ship to verify proper operation after deployment.

A single CMOS IM6100 microprocessor performs both control and signal-processing functions in the receiver (Fig. 8). Signal acquisition is initiated and signal processing performed as in the WHOI receivers, except that conventional analog-to-digital converters are used to digitize the quadrature components to 12 bit (Fig. 9). Sample-and-hold circuits ensure that simultaneous samples are acquired for all four hydrophones. After "sharp" processing, the 32 largest peaks in the arrival pattern are located and five samples centered on each peak are saved on tape.

A two-oscillator system analogous to that employed in the acoustic sources is used to achieve the required submillisecond timing precision. A low-power $(200-\mathrm{mW})$ ovenized crystal oscillator (OXO) runs continuously, and is compared at 12-h intervals with a Rb atomic frequency standard to one part in $10^{10}$. A digital frequency comparison technique is employed, in contrast to the partly analog phase-comparison technique implemented in the sources. The $10-\mathrm{MHz}$ signal supplied by the $\mathrm{Rb}$ oscillator is doubled to $20 \mathrm{MHz}$ using a monolithic phase-lock loop, gated for $500 \mathrm{~s}$ using the $1-\mathrm{MHz}$ OXO output, and counted. No feedback technique is employed to adjust the OXO frequency since it is inherently more stable than the TCXO used in the sources by approximately one order of magnitude (Fig. 5). Overall performance of the clockcorrection system was comparable to that achieved in the WHOI sources; corrected instrument time departed from UTC by less than $1 \mathrm{~ms}$ at the end of the experiment.

A number of features are included in the SIO receivers to increase the reliability of these instruments. A low-power (10$\mathrm{mW}$ ) temperature-compensated crystal oscillator with its own timing chain provides backup for the ovenized crystal oscillator and primary clock chain. Data are recorded on four tape recorders used in rotation, with successive receptions written to different recorders. Failure of a recorder therefore results in the loss of every fourth reception instead of the loss of data from one-quarter of the experiment days, as would happen if the magnetic tapes were filled sequentially. High-frequency acoustic command and data links are provided between the receiver and the ship to verify proper operation after deployment. These systems are described in more detail below.

A number of diagnostics are performed to aid in assessing data quality at the conclusion of the experiment. For example, in conjunction with each acoustic reception, the proper functioning of the amplifier, demodulator, and analog-todigital converter chains is tested by injecting calibration signals into the input of each analog receiver and measuring the resulting signal levels. Ambient noise levels are measured independently for each hydrophone channel to verify that they are consistent. Basic system integrity is monitored at regular intervals by measuring system voltages and currents, by performing RAM tests, by forming checksums of the EPROM's containing the program, and by intercomparing the two clock chains.

\section{B. Acoustic Command and Data Links}

The SIO receivers include high-frequency acoustic data links for in situ communications with shipboard. The basic goal is to verify proper operation after deployment. Only a limited capability to command the capsule from shipboard is therefore implemented. A standard Interocean acoustic command receiver identical to that employed in their releases is 


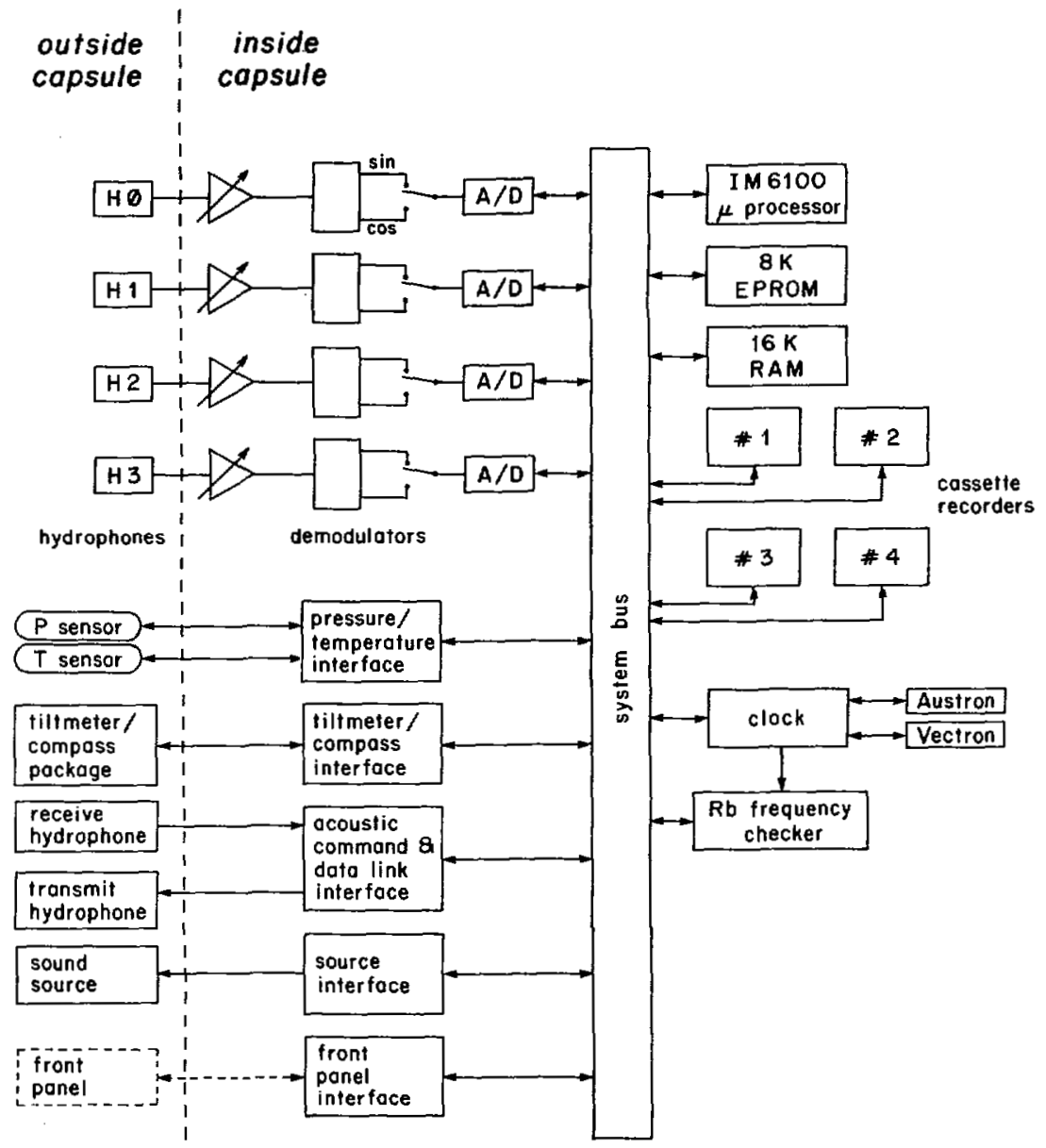

Fig. 8. Block diagram of the SIO receiver electronics. The connection shown by the dashed line between the front panel and the receiver is not present in the water.

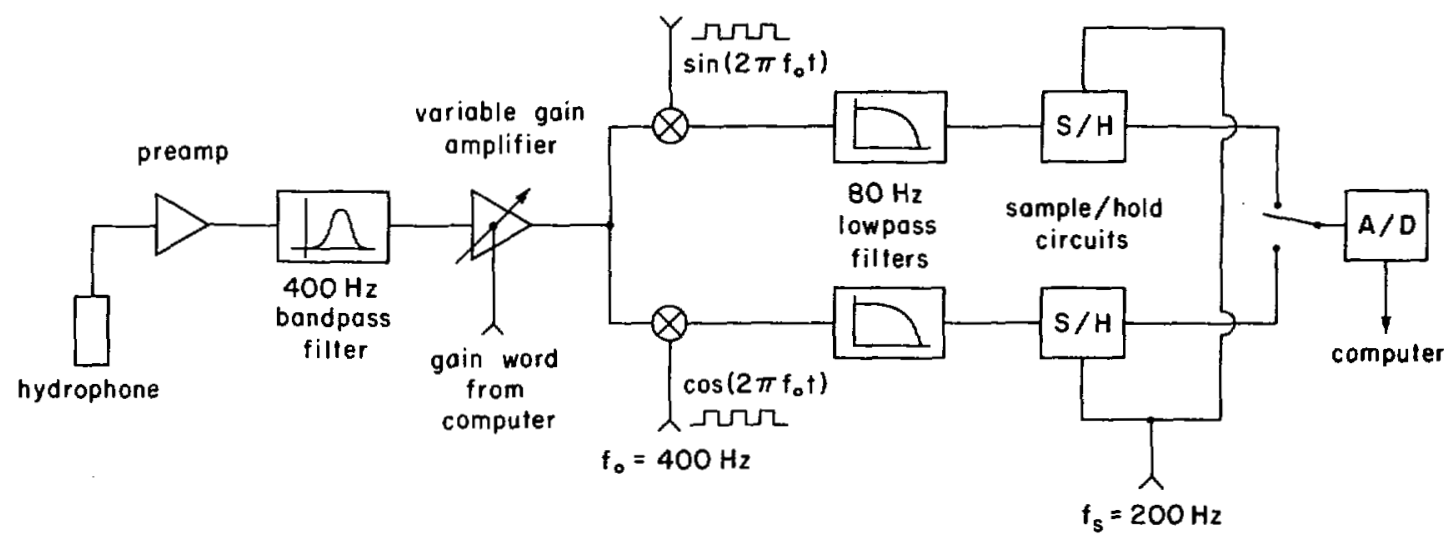

Fig. 9. Block diagram of the analog square-wave demodulator employed in the $S I O$ receivers.

installed in the SIO units. The Interocean receiver requires that exactly four out of eight frequencies between 14 and $16 \mathrm{kHz}$ be present for a valid command; a total of 70 commands are therefore available. A two-part sequence, consisting of an address followed $25 \mathrm{~s}$ later by a command, is used to maximize the number of available commands.

A frequency-shift-keyed (FSK) digital data link is used to relay receiver status information to the ship to verify proper operation. A novel approach is employed to both eliminate multipath interference effects and free the ship to continue operations in the vicinity of the transceiver mooring while monitoring the data link. The FSK signal is transmitted at 600 Bd in short bursts of approximately $0.25 \mathrm{~s}$ at $15-\mathrm{s}$ intervals. It is received on a standard U.S. Navy sonobuoy with a $1000-\mathrm{ft}$ hydrophone depth and radioed to shipboard. If the sonobuoy is directly above the mooring, there exists a 0.4 -s period during which the signal arriving via the direct path is free of multipath interference effects. As the sonobuoy drifts away from the 


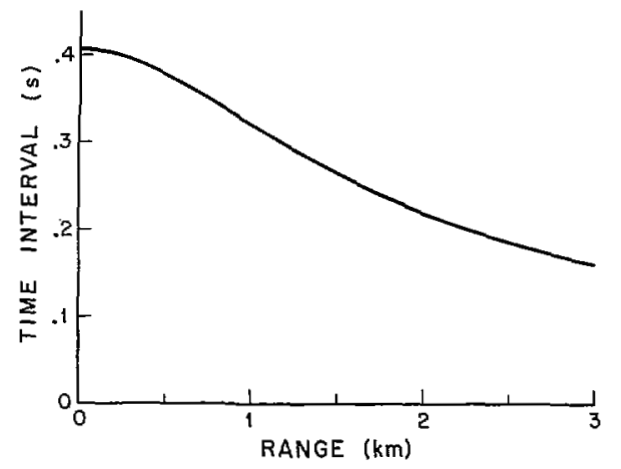

Fig. 10. Time interval between the direct and surface-reflected arrivals at the sonobuoy hydrophone as a function of horizontal range. The source is assumed to be at a depth of $1300 \mathrm{~m}$.

mooring, the interference-free time steadily decreases, until the signal begins to be garbled and it becomes necessary to deploy another sonobuoy (Fig. 10). Our experience has been that one sonobuoy has provided adequate communications for several hours under typical midocean conditions, but this would not be the case in the presence of strong ocean currents. Frequencies of 7 and $8 \mathrm{kHz}$ are employed for the FSK link; we have found that somewhat higher frequencies would be desirable to avoid interference from ship-generated noise at these relatively low frequencies.

\section{Vertical-Array Performance}

The vertical hydrophone arrays provide information on the vertical arrival angles at the receivers, provide improved signal-to-noise ratios, and permit the separation in angle of some arrivals that are not resolved in the time domain [22]. Vertical arrival-angle information assists in ray identification, and is potentially useful in performing inversions to convert travel-time differences to ocean-current structure. The hydrophones in the vertical arrays are separated by $(3 / 2) \lambda$, where $\lambda$ is the acoustic wavelength. The resulting anguiar resolution (defined as the ability of an array to separate simultaneous arrivals from different angles) is approximately $9^{\circ}$, as measured by the 3-dB full beamwidth (Fig. 11). Secondary beams that exist at high angles (because the element spacing is greater than $\lambda / 2$ ) do not cause ambiguities since all signals arrive within $\pm 15^{\circ}$ of horizontal. The angular precision (defined as the ability of an array to measure the arrival angle of an isolated arrival) is given by a formula analogous to (1)

$$
\sigma_{\phi}=\left(\beta_{x} \sqrt{2 E / N}\right)^{-1}
$$

where $2 E / N$ is the signal-to-noise ratio. Also

$$
\beta_{x}=2 \pi\left[\int_{-\infty}^{\infty} x^{2} I(x) d x\right]^{1 / 2}
$$

is the rms length of the antenna illumination function $I(x)$, and $x$ is in wavelengths [23]. Four discrete elements separated by $d / \lambda$ give $\beta_{x}=\sqrt{5} \pi(d / \lambda)$. With $d / \lambda=3 / 2$ and $25-\mathrm{dB}$ signalto-noise ratio, one obtains $\sigma_{\phi}=0.0053 \mathrm{rad}$, or $0.3^{\circ}$, which is adequate for ray identification.

True time-delay beamforming of the complex demodulates

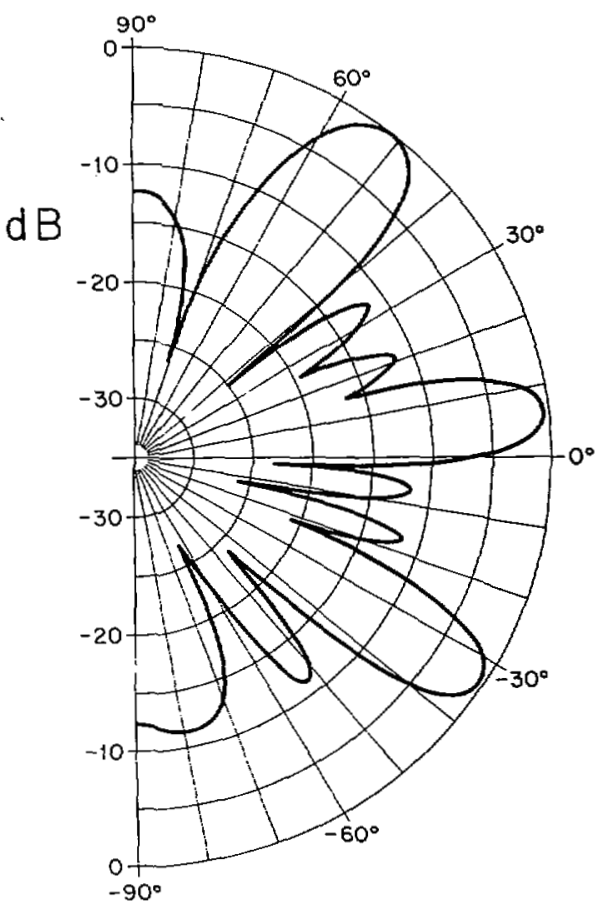

Fig. 11. Computed beam pattern for the SIO vertical hydrophone array. The main beam has been steered to $+6.5^{\circ}$ relative to the horizontal in this example.

has been implemented [24]; the signal bandwidth is too great to permit the application of simple phase-shift beamforming. Since the acoustic data were processed in situ and amplitude and phase information retained only for samples within two sample intervals of the times of the arrival peaks, it is impossible to implement the interpolation required for timedelay beamforming using standard digital-interpolation techniques. Simple parabolic fits to three points centered on the arrival peaks have therefore been used to separately interpolate the real and imaginary parts of the complex demodulates prior to beamforming. The adequacy of this technique has been evaluated using data obtained during a preliminary equipment test conducted during 1982 that had a geometry similar to that utilized in the 1983 Reciprocal Transmission Experiment. One instrument was programmed to record the entire received signal, rather than just peak information, so that standard digital-interpolation techniques could be used (periodic sinc interpolation). Arrival angles measured for several different ray paths using the two techniques agreed in the mean to a few hundredths of a degree, and the standard deviation of the differences was approximately $0.5^{\circ}$. The standard deviation of the differences is close to the theoretically predicted arrivalangle precision, and is therefore acceptable.

The signal-to-noise ratio improvement provided by the vertical array can be found by comparing the signal-to-noise ratio achieved with a single hydrophone for a selected resolved arrival with that achieved with an appropriately steered array. Since the acoustic data were processed in situ and amplitude and phase information retained only at the times of the arrival peaks, it is impossible to evaluate the effect of beamforming on the ambient noise for the 1983 Reciprocal Transmission Experiment. During the preliminary experiment conducted in 
TABLE П

ARRAY GAINS MEASURED FOR FOUR RAYS DURING A PRELIMINARY EXPERIMENT CONDUCTED IN 1982

\begin{tabular}{|c|c|c|c|}
\hline Path & Nominal & Array & Number of \\
\hline \multirow[t]{2}{*}{ Identifier } & Arrival Angle & Gain & Samples \\
\hline & & $(\mathrm{dB})$ & \\
\hline$+15(896 \mathrm{~m})$ & $-7.1^{\circ}$ & $6.7 \pm 1.1$ & 10 \\
\hline$-13(860 \mathrm{~m})$ & $+7.9^{\circ}$ & $7.0 \pm 1.0$ & 11 \\
\hline$+13(787 \mathrm{~m})$ & $-9.1^{\circ}$ & $6.2 \pm 1.0$ & 8 \\
\hline$-11(731 \mathrm{~m})$ & $+9.8^{\circ}$ & $6.4 \pm 0.7$ & 11 \\
\hline
\end{tabular}

The array gains are the differences between the average signal-to-noise ratios for the four individual hydrophones and the signal-to-noise ratios obtained with beams pointed in the nominal ray arrival directions. The observed standard deviations in array gain are also given.

1982, however, the entire signal recorded by one instrument included ambient noise. The array gains measured for four well-resolved paths in the 1982 data (Table II) are consistent with the value of $6 \mathrm{~dB}$ predicted for our array in the presence of isotropic noise [14]. Previous measurements of ambientnoise directivity at $400 \mathrm{~Hz}$ are limited [25], [26], but indicate that an assumption of isotropic noise is reasonable for moderate sea states (Sea State 3).

Vertical arrival angles measured at the SIO receiver on the North mooring in 1983 were found to be consistent with theoretical predictions for every case examined (Table III). The arrival angles were computed at the arrival times of eight well-resolved ray paths during a 10-day period when the mooring tilt was measured to be less than $1^{\circ}$ (year days 220 230). These results confirm the ray identification made in Fig. 3.

\section{Signal and Noise Levels}

The receiver gain in the SIO transceivers was digitally adjusted using multiplying digital-to-analog converters; all four hydrophone channels utilized the same gain. The receivers sampled the ambient-noise field prior to each reception to determine its level, and then set the gain such that the peak-topeak noise would be one-eighth of full scale. Since the received signal level prior to signal processing is predicted to be well below the ambient-noise level, this ensures that the receivers will not saturate when sampling the signal (the number of times that the analog-to-digital converters were saturated was recorded for each reception to verify that the system was functioning properly). The measured noise levels, the gain at which they were measured, and the gain used for the signal reception were all recorded on tape for later analysis. It is therefore possible to determine the absolute levels of the ambient noise and the received signal at the SIO transceivers for comparison with the predictions given in Table I. Fig. 12 gives the rms signal level prior to signal processing for path $B$ (Fig. 3), the ambient-noise level, and the signal-to-noise ratio after processing. The signal level is found to be roughly $10 \mathrm{~dB}$ below that predicted for spherical
TABLE III

THEORETICAL AND MEASURED ARRIVAL ANGLES FOR THE SIO RECEIVER ON THE NORTH MOORING. THE MEASURED ARRIVAL ANGLES WERE DETERMINED FROM A HISTOGRAM OF OBSERVED ARRIVAL ANGLES WTTH $0.5^{\circ}$ BIN WIDTH

\begin{tabular}{ccc}
\hline \multicolumn{2}{c}{ Ray path } & \multicolumn{2}{c}{$\frac{\text { Arrival Angle }}{\text { Measured }}$} & $\frac{\text { Predicted }}{\text { (degrees) }}$ & (degrees) \\
$-8(123 \mathrm{~m})$ & -11.75 & -11.66 \\
$+9(98 \mathrm{~m})$ & -12.25 & -12.05 \\
$+11(617 \mathrm{~m})$ & -11.75 & -11.12 \\
$+12(778 \mathrm{~m})$ & +9.50 & +9.72 \\
$-12(782 \mathrm{~m})$ & -10.00 & -9.68 \\
$+13(812 \mathrm{~m})$ & -9.75 & -9.32 \\
$-13(882 \mathrm{~m})$ & +7.75 & +8.30 \\
$+15(927 \mathrm{~m})$ & -7.25 & -7.50 \\
\hline
\end{tabular}

spreading. Since path $B$ is smaller than the largest arrival by a comparable amount, the highest peak in the received signal is approximately spherically spread. The ambient-noise level is usually below that predicted for Sea State 3 . The noise is typically within a $10-\mathrm{dB}$ band for the duration of the reciprocal transmissions, although large noise spikes (presumably due to ships passing near the mooring) are occasionally present. The resulting signal-to-noise ratio is usually somewhat below the nominal value of $24.9 \mathrm{~dB}$ predicted for spherical spreading. It will of course be larger for the stronger arrivals.

While the SIO receivers do provide absolute signal and noise levels, this was not their primary function; they have therefore not been calibrated with the care expected for absolute ambient-noise level measurements. The absolute source level is also not well known. The accuracy of these results is therefore difficult to determine, but is probably close to $\pm 3 \mathrm{~dB}$. The relative accuracy for measurements made at different times is much higher since the components of the system are quite stable.

\section{MOORING MOTION}

Motions of the moored instruments change the physical path lengths of the acoustic rays, perturbing the measured travel times; a 150-m path-length change causes a travel-time change of $100 \mathrm{~ms}$. Mooring excursions of hundreds of meters in response to barotropic tidal currents and currents associated with the ocean eddy field are common even for the taut [1500lb $(\sim 6.7-\mathrm{kN})$ tension] moorings used in this experiment. When the acoustic transmissions are in one direction only, as in the 1981 Ocean Acoustic Tomography Experiment, these substantial travel-time changes are intertwined with those due to sound-speed fluctuations along the transmission path. In order to separate the effects of mooring motion from oceanic variability, it is necessary to measure continuously the relative 

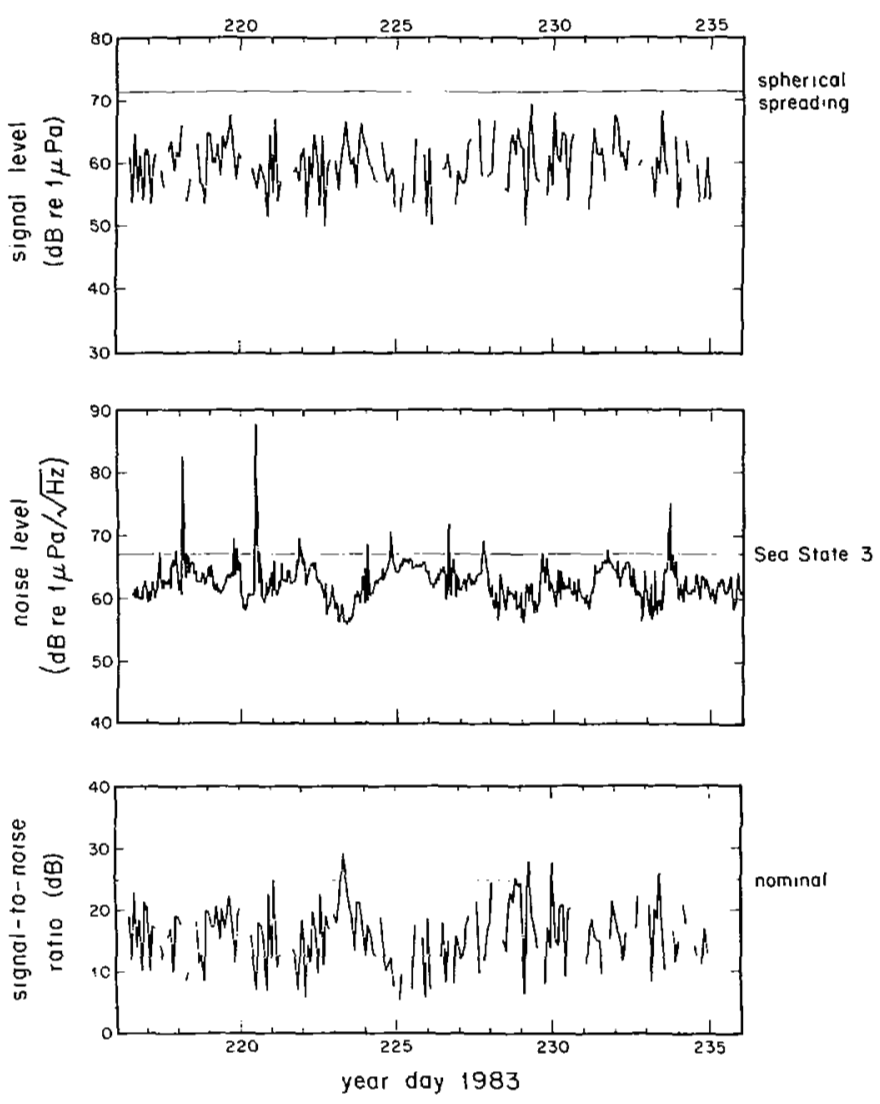

Fig. 12. Absolute signal and noise levels, and the resulting signal-to-noise ratio after processing, at the SIO receiver on the North mooring. The rms signal level is for path $B$ (Fig. 3); gaps correspond to times when this path was not among those for which information was stored. The signal level corresponding to spherical spreading, the predicted Sea-State 3 noise level, and the nominal signal-to-noise ratio from Table I are indicated. The receiver bandwidth and the processing gain are required to obtain the signal-to-noise ratio from the absolute signal and noise levels.

displacement of the instruments, or to consider instrument displacement as an unknown in the mathematical inversion procedure through which travel times are converted into estimates of the sound-speed field. This latter procedure introduces additional unknowns in the inversions, and therefore has not been an a priori part of any experiments to date.

In the case of reciprocal transmissions, the change in path length between transceiving instruments is less important. The differential travel time is directly proportional to the rayaveraged current with respect to the mean motion of the transceivers [1]. An error in the assumed range between transceivers alters the estimation of mean ocean current only by a factor of $[1-\delta R / R]$, where $R$ is the transceiver separation and $\delta R$ is the path-length change. For $\delta R=1 \mathrm{~km}$ and $R=300 \mathrm{~km}$, this gives 0.3 -percent error. However, since mooring motion has a strong tidal component, it is necessary to correct the measured currents for the mooring velocities to obtain accurate tidal estimates. The motions of the moored instruments in this experiment were measured with this intent. In addition, knowledge of mooring motion allows for inversion of one-way travel-time data, and it assists in the forward modeling process (ray-path identification) where an accurate knowledge of path lengths is required.
A pulsed acoustic navigation technique is employed to track the position of the instruments [10]. Three transponders are deployed on the seafloor in an equilateral triangle about the base of the mooring at a distance from the mooring anchor approximately equal to the height of the transceiver off the bottom (about $3700 \mathrm{~m}$ ). An acoustic interrogator is attached to the mooring within $50 \mathrm{~m}$ of the transceivers. The interrogator measures the round-trip travel times of acoustic pulses $(10 \mathrm{~ms}$ long, in the $10.5-13.5 \mathrm{kHz}$ band) from the interrogator to the transponders every $15 \mathrm{~min}$. Slant ranges are calculated, and the motion of the interrogator, which is essentially the same as the transceiver, is calculated in cartesian coordinates. The overall accuracy of the system is estimated to be a few meters.

The motion of the transceiver on the North mooring is shown in Fig. 13. The mooring moves in excess of $1000 \mathrm{~m}$ in the horizontal during the 20-day period. Ocean currents at $1000-\mathrm{m}$ depth on the mooring and the tilt of the mooring at the depth of the transceiver are also shown. As expected, the mooring responds to the current field. It is initially displaced to the northeast, and then gradually relaxes back to near vertical as the flow approaches zero around day 223. Beginning about day 231, the mooring again undergoes substantial displacements, with sharp reversals in the current components on day 234 reflected in the mooring displacements and tilts. The maximum tilts observed at the depth of the transceiver are in excess of $4^{\circ}$. The mooring behavior and current records after day 231 apparently reflect the passage of an energetic mesoscale feature past the North mooring. In addition to this gross motion, tidal variations of about 2 cycles per day are present in both the mooring motion and current data.

\section{PRELIMINARY RESULTS}

The instrumentation described above was deployed during August and September of 1983 to the west of Bermuda. The data have been analyzed to yield the differential travel times of identified arrivals, but the techniques of inverse theory have not yet been employed to derive the range-averaged sound speed and current fields.

\section{A. One-Way Transmissions}

Daily-averaged arrival amplitudes for the SIO receiver on the North mooring are very similar from day to day (Fig. 14); the amplitudes are roughly constant. The variation in total travel time is due primarily to changes in the source-receiver range because of mooring motion. A 0.75 -s change implies a differential horizontal mooring displacement of approximately $1100 \mathrm{~m}$, roughly consistent with the measured mooring motion.

In order to perform an inversion of travel-time data, each arrival must be associated with a particular ray path. This identification is achieved by comparing predicted and measured receptions. In Fig. 3 the daily average for day 217 is compared with a WKBJ prediction. The correspondence is excellent, except for the final cluster of near-axial arrivals (probably because the WKBJ method is not valid for near-axial rays). 

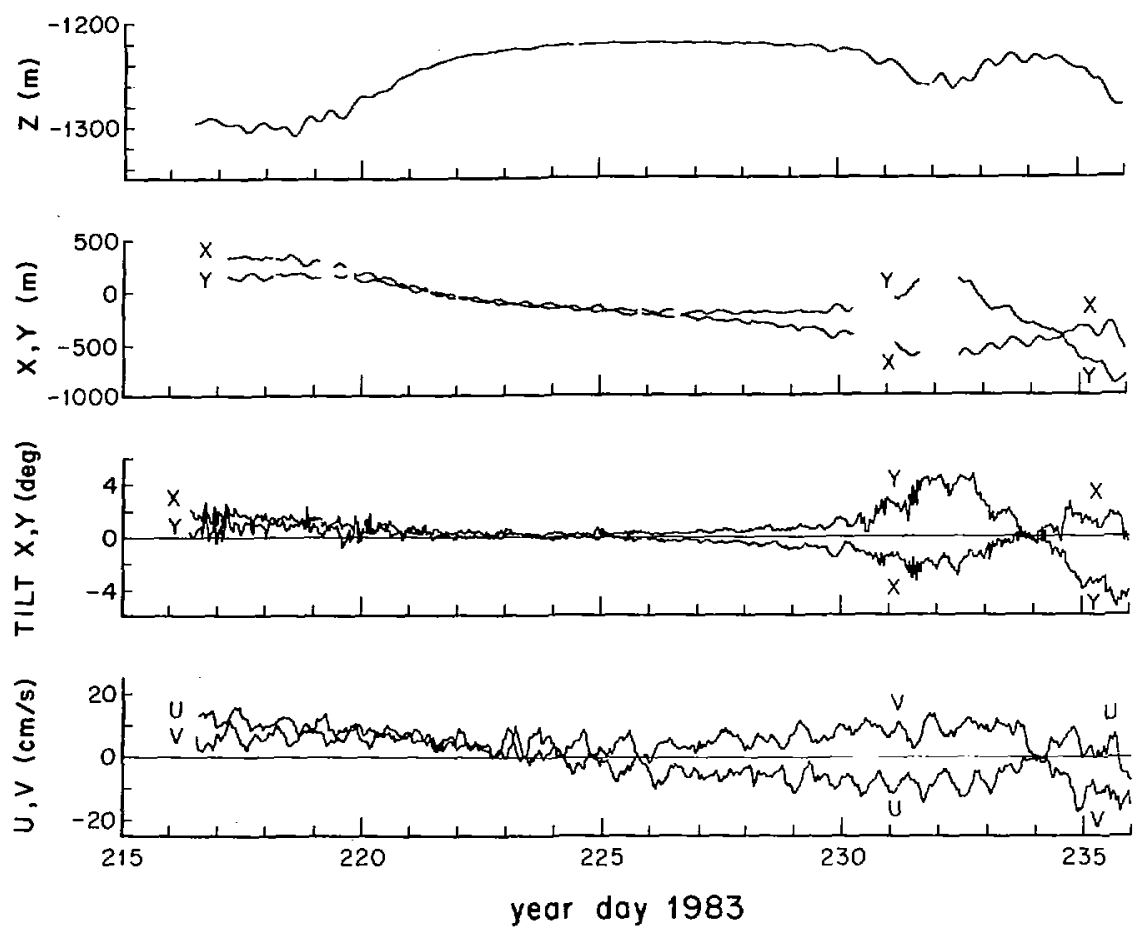

Fig. 13. Transceiver position, mooring tilt, and ocean currents at the North mooring. The top panel displays the SIO transceiver depth as measured by a pressure sensor attached to it. The second panel shows the east $(x)$ and north $(y)$ excursions of the transceiver as measured by the acoustic navigation system. Gaps are due to missing data. The third panel shows the east $(x)$ and north $(y)$ tilt of the mooring at the depth of the transceiver. The east $(u)$ and north $(v)$ current components at 1000-m depth are displayed in the bottom panel.

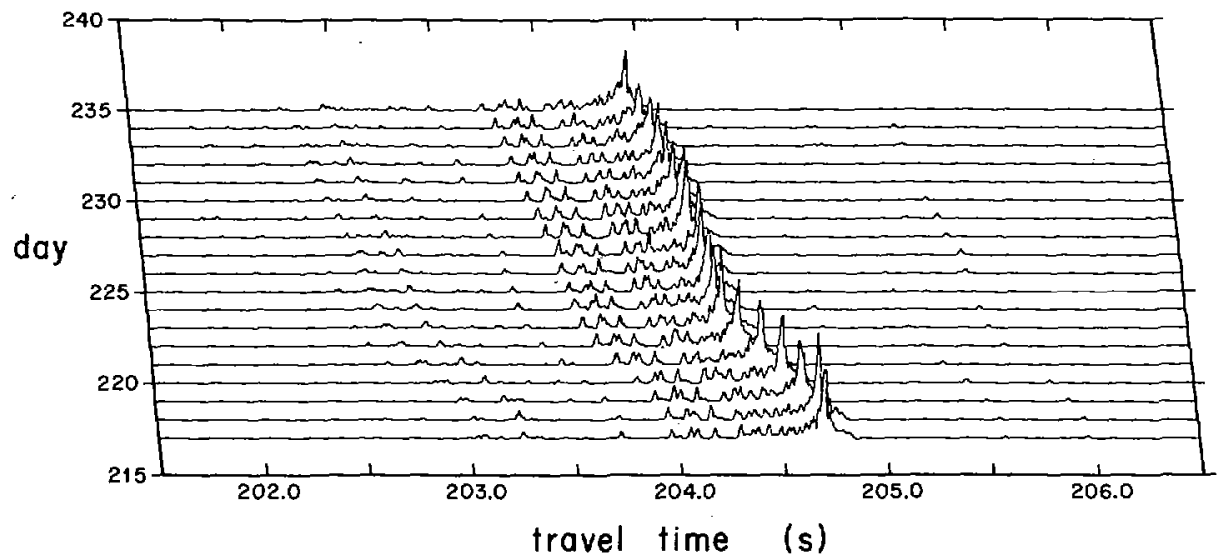

Fig. 14. Daily-averaged arrivals for the SIO receiver on the North mooring. Path $A$ was used to align the bihourly receptions prior to averaging.

\section{B. Reciprocal Transmissions}

Fig. 15 shows a sequence of reciprocal arrival patterns. The number of arrivals and the spacing between arrivals are quite similar. The amplitudes, though, vary as much as a factor of two (see Fig. 14), and at times an arrival can disappear entirely. This may be a result of retaining only the 32 highest peaks (thus losing small ones) or it may be due to ocean changes. Internal waves can cause the basic ray path to split into micromultipaths which then interfere at the receiver, sometimes destructively [27], [28]. Daily averages suppress internal wave-related fluctuations, and the daily-average arrival patterns for day 223 are almost identical except for the cluster of arrivals near $204.25 \mathrm{~s}$ (from near-axial paths). Interference effects make this part of the pattern very sensitive to small differences. The fact that the amplitudes are different for the two receivers indicates that nonreciprocity due to currents and current shears is present.

\section{Interpolation}

The theoretical arrival-time precision is approximately 0.3 $\mathrm{ms}$, while the sample interval is $5 \mathrm{~ms}$. It is therefore necessary 


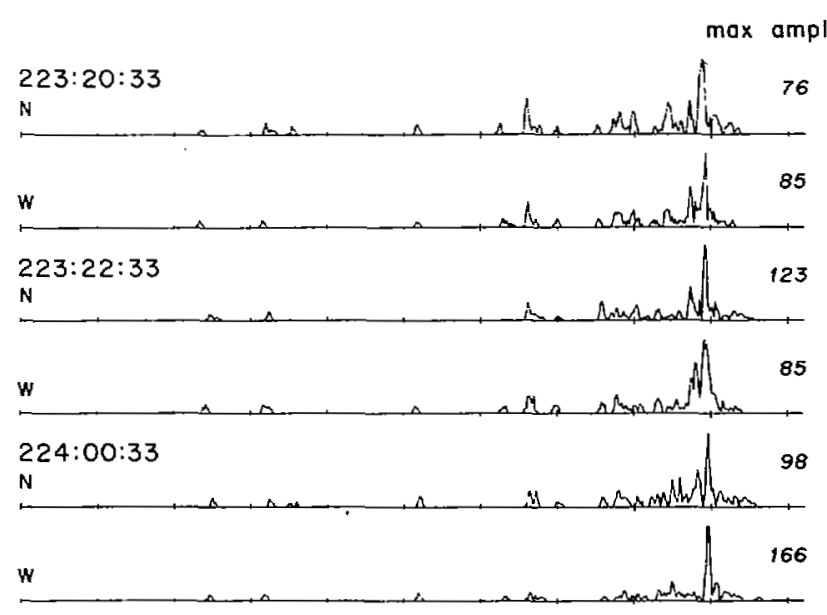

(a)

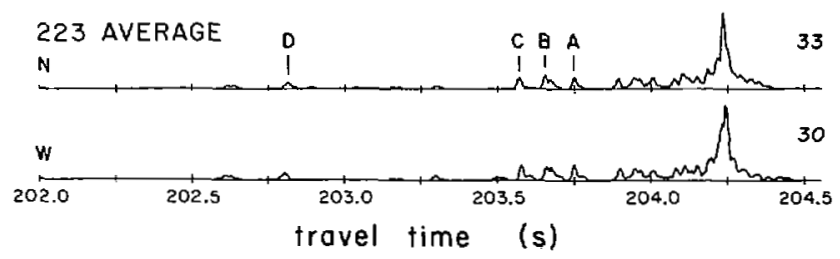

(b)

Fig. 15. Arrival amplitudes of reciprocal receptions by the SIO receivers on the North and West moorings: (a) three pairs of bihourly receptions starting at 2033 GMT on day 223, and (b) daily averages for day 223 . The vertical scales are proportional to pressure. Path $A$ has been used as reference.

to interpolate the available data to accurately determine arrival peak locations. If complete reception records had been saved (rather than only information on the peaks), a reasonable technique to employ would be periodic sinc interpolation, since the data are periodic and reasonably band-limited. With only a few samples available to define the peaks, this is not possible and much simpler algorithms that fit parabolic and Gaussian curves to three adjacent points have been used.

The simple curve-fitting algorithms were evaluated using computer simulations and laboratory data. The errors incurred are a function of the relative locations of the data samples and the arrival peak. The rms errors obtained from many different trials show that parabolic and Gaussian interpolation yield travel times accurate to within a few percent of the sample interval for the ideal signals considered here (Table IV). Table IV also displays the rms errors incurred by simply using the largest sample to determine the arrival time and by using cubic spline interpolation (which requires the complete reception record).

Based on these results, Gaussian interpolation has been selected to determine travel times. The expected rms error in travel time is about $0.03 \Delta t$ or $0.15 \mathrm{~ms}$. More sophisticated techniques are not justified until the signal-to-noise ratio is increased and the actual peak shape, a function of both the source and the ocean sound channel, is known.

\section{Correcting One-Way Travel Times}

The raw travel times must be corrected for mooring motion and clock error before they can be used. Fig. 16 displays the
TABLE IV

RMS TRAVEL-TTME ERRORS FOR DIFFERENT INTERPOLATION SCHEMES. VALUES SHOWN ARE PERCENTAGES OF $\Delta t$, THE SAMPLE INTERVAL

\begin{tabular}{lcc}
\hline & Simulation & Lab Test \\
\hline $\begin{array}{l}\text { No interpolation } \\
\text { (maximum point) }\end{array}$ & 29.3 & 28.3 \\
Parabola & 4.9 & 4.3 \\
Gaussian & 2.3 & 2.7 \\
Cubic spline & 4.3 & 3.5 \\
\hline
\end{tabular}

raw travel times for path $C$, the corrections due to mooring motion and clock error, and the final corrected travel times. The convention here is that the corrected travel time equals the uncorrected value minus the corrections.

The mooring-motion correction refers the measured travel time to a fixed reference ray path using the plane-wave approximation. This removes the effect on travel time of relative source receiver displacements. This correction is calculated using the position of the interrogators as a function of time (Fig. 13), the nominal vertical separations of instruments on the moorings, the tilts of the moorings, the ray-path headings at each mooring, the ray angles in the vertical, and the local sound speeds. In using the mooring tilt to better define instrument position, we assume the mooring is linear in the instrument section. Most of the change in raw travel time is due to the source and receiver moving together about $1100 \mathrm{~m}$. For one-way travel times, the mooring-motion correction is most important.

The clock corrections over the time span plotted are at most 24 ms. An additional timing correction, a constant, must be applied to account for electronic delays in the source and receiver and the delay from the nominal beginning of the source transmission to the receiver sample time. The applied constant correction for this case is $-199.9791 \mathrm{~s}$.

When the net mooring-motion correction (Fig. 16(b)), the clock corrections (Fig. 16(c)), and the constant timing correction are added together and subtracted from the raw travel times, we obtain the corrected travel-time series (Fig. 16(d)). Note that the trend in the corrected data is the reverse of that in the uncorrected data. There is a roughly linear change of $0.15 \mathrm{~s}$ in travel time over the 19 days shown. This corresponds to a $1.1-\mathrm{m} / \mathrm{s}$ change in sound speed, or a change of $0.2^{\circ} \mathrm{C}$, along the ray path. The high-frequency fluctuations could be due to internal waves or measurement noise.

\section{E. Differential Travel Times}

Differential travel time $\Delta$ is, to first order, related to the velocity component along the ray path $\Gamma$ by

$$
\Delta=\frac{2}{C_{0}} \int_{\Gamma} \frac{u}{C_{0}} d s \sim 2 \frac{u}{C_{0}} \frac{R}{C_{0}}
$$

where $C_{0}$ is a reference sound speed, $s$ is the arc length along 


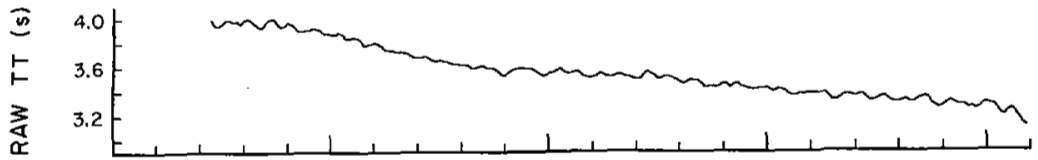

(a)

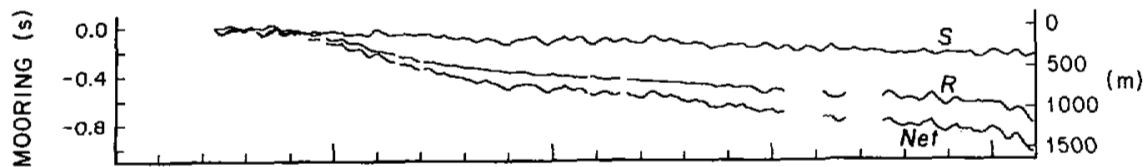

(b)

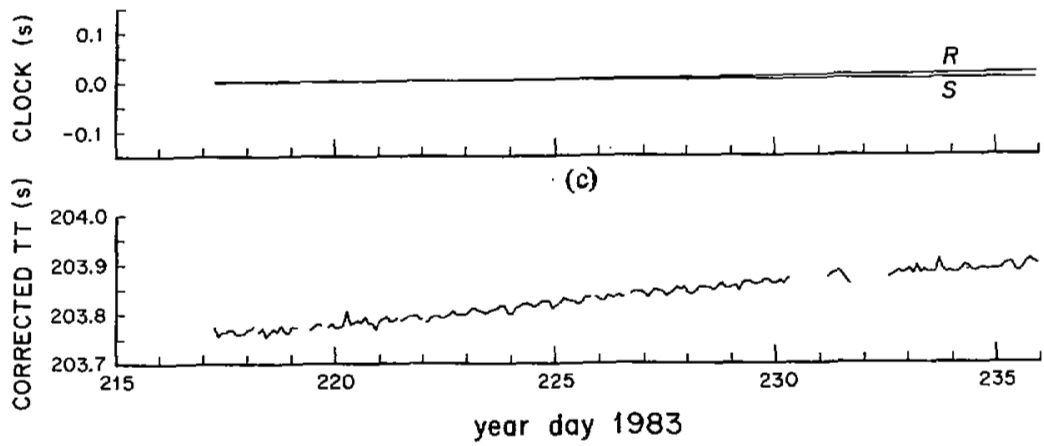

(d)

Fig. 16. Correcting path $C$ one-way travel times. (a) Shows the raw tracked peak series. (b) Shows the corrections for source motion $S$, receiver motion $R$, and the net motion of the two. The right-hand vertical axis of (b) shows the equivalent displacement along the ray path. (c) Shows the source and receiver clock corrections. (d) Shows the final corrected traveltime series. This is equal to the raw travel time minus the corrections in (b) and (c) and a constant time correction explained in the text. Note that the vertical scales of panels (c) and (d) are expanded by a factor of four relative to panels (a) and (b).

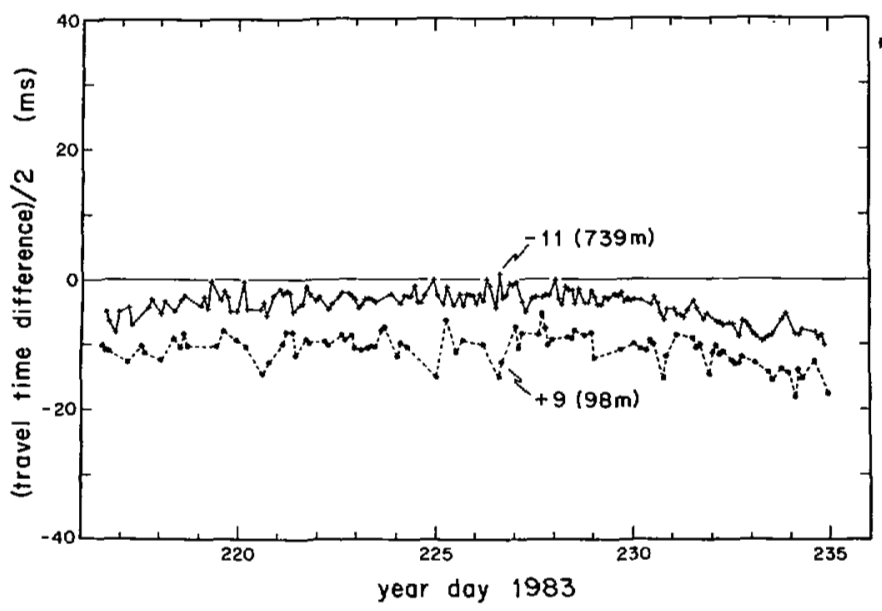

Fig.17. Differential travels times $\Delta / 2$ for Rays $C$ and $D$. $\Delta$ is the travel time measured from North to West minus that measured from West to North.

the ray path, and $R$ is the range between the transceivers. The greatly simplified expression on the right is obtained by assuming the velocity is constant over the path. We can use this simplified expression as a rough guide in interpreting differential travel-time data in terms of velocity.

Time series of differential travel time are shown for two rays in Fig. 17. Ray $C$ has an upper turning point at $739 \mathrm{~m}$ and ray $D$ has one at $98 \mathrm{~m}$. The data have been corrected for clock drift and time delays in the system. The received signals have been referenced to the source locations using the plane-wave approximation and the nominal ray arrival angle, removing differential travel times caused by the (small) vertical separations of the sources and receivers. Mooring motion has been neglected. Its only effect is that the measured velocity is relative to the mean velocity of the moorings during the actual transmission.

A rough value of velocity (positive in the direction from West to North mooring) in centimeters per second can be obtained (using the simplified expression) by multiplying $\Delta / 2$ $\mathrm{ms}$ in the figure by $C_{0}{ }^{2} / R=0.75$. The mean differential travel times for paths $C$ and $D$ are $-3.9 \mathrm{~ms}$ and $-9.8 \mathrm{~ms}$, respectively. The corresponding mean current velocities of $-2.9 \mathrm{~cm} / \mathrm{s}$ and $-7.4 \mathrm{~cm} / \mathrm{s}$ are reasonable, and the direction is consistent with Gulf Stream recirculation. Ray $D$ indicates a larger velocity closer to the surface than ray $C$ turning at a greater depth, also consistent with expectation. A lowfrequency trend is evident in the data, with the differential travel times somewhat more negative near the beginning and end of the experiment. The long-term variation is most likely a result of the passage of a mesoscale feature. The highfrequency variation is probably due to tides and internal waverelated fluctuations.

\section{DISCUSSION}

The preliminary results given above are promising. The differential travel times have reasonable magnitudes and a low-frequency trend that is presumably caused by mesoscale fluctuations in the ocean.

One of the unique advantages to using acoustic techniques to measure large-scale oceanic phenomena is that acoustic 
propagation inherently gives an integral or spatially averaged result. For many purposes it is spatial averages that are of interest, and these are extremely difficult to obtain over large ocean areas in any other way. Much work remains to relate the measured differential travel times to meaningful ocean currents, however. One potential source of error in the inversion of differential travel times to obtain currents is the fact that current shear causes ray paths with and against a current to differ. Sound pulses traveling in opposite directions therefore do not sample precisely the same part of the ocean. Fermat's principle states, however, that travel time is unchanged to first order in small path perturbations. Effects associated with the nonreciprocity of ray paths are therefore expected to be small in most situations. It is, however, possible to conceive of situations where this is not so [29]. If the sound-speed gradient is much less than the current gradient in some part of the water column, then the current gradient will dominate in that region and cause ray paths with and against the current to be well separated. More study is required to fully understand just how important this problem is likely to be. It will certainly be necessary to avoid rays that are sensitive to ray-path nonreciprocity when measuring differential travel times. After inverting for the current field from the differential travel times, it may be adequate to perform an a posteriori sensitivity test by tracing rays for the combined sound-speed and current fields.

\section{ACKNOWLEDGMENT}

The authors wish to thank F. Dormer, K. Hardy, B. Ma, D. Peckham, and R. Truesdale of Scripps Institution of Oceanography and P. Boutin, A. Bradley, J. Kemp, S. Liberatore, K. Peal, S. Rosenblad, and F. Schuler of Woods Hole Oceanographic Institution for their attention to instrument design, fabrication, software development, testing, maintenance, and at-sea deployment. It is impossible to imagine more professional and dedicated teammates and much of the credit for successful system operation should be theirs. D. Webb designed and built the acoustic sources. J. Spiesberger analyzed the mooring-motion data presented here. T. Birdsall and $K$. Metzger are in large measure responsible for the signal-processing techniques used in this experiment. B. Cornuelle developed the program used to track the acoustic arrivals. Xu Yukun of the People's Republic of China aided in the development of the in situ source recording system. G. Williams was responsible for the development of an antecedent to the current generation of SIO receivers. W. Munk and C. Wunsch provided advice and encouragement in all aspects of the work.

\section{REFERENCES}

[1] P. Worcester, "Reciprocal acoustic transmission in a midocean environment," J. Acoust. Soc. Amer., vol. 62, pp. 895-905, Oct. 1977.

[2] W. Munk and C. Wunsch, "Ocean acoustic tomography: A scheme for large scale monitoring," Deep-Sea Res., vol. 26, pp. 123-161, Feb. 1979.

[3] The Ocean Tomography Group, "A demonstration of ocean acoustic tomography," Nature, vol. 299, pp. 121-125, Sept. 1982.

[4] B. Cornuelle, C. Wunsch, D. Behringer, T. Birdsall, M. Brown, R. Heinmiller, R. Knox, K. Metzger, W. Munk, J. Spiesberger, R. Spindel, D. Webb, and P. Worcester, "Tornographic maps of the ocean mesoscale-1: Pure acoustics," J. Phys. Oceanogr., to be published.

[5] P. Worcester and B. Cornuelle, "Ocean acoustic tomography: Cur- rents," in Proc. IEEE Second Working Conf. Current Measurements, Nov. 1982, pp. 131-134.

[6] W. Munk and C. Wunsch, "Up-down resolution in ocean acoustic tomography," Deep-Sea Res., vol. 29, pp. 1415-1436, 1982.

[7] T. Rossby, "An oceanic vorticity meter," J. Mar. Res., vol. 33, pp. 213-222, 1975

[8] W. Munk and C. Wunsch, "Observing the ocean in the 1990's," Phil. Trans. Roy. Soc. London A, vol. 30, pp. 439-464, 1982.

[9] M. Longuet-Higgins, "On triangular tomography," Dynam. Atmos. Oceans, vol. 7, pp. 33-46, 1982.

[10] R. Spindel, P. Worcester, D. Webb, P. Boutin, K. Peal, and A. Bradley, "Instrumentation for ocean acoustic tomography," in Oceans 82 Conf. Rec., Sept. 1982, pp. 92-99.

[11] C. Chen and F. Millero, "Speed of sound in seawater at high pressures," J. Acoust. Soc. Amer, vol. 62, pp. 1129-1135, Nov. 1977.

[12] M. G. Brown, "Application of the WKBJ Green's function to acoustic propagation in horizontally stratified oceans," J. Acoust. Soc. Amer., vol. 71, pp. 1427-1432, June 1982.

[13] W. Munk and C. Wunsch, "Ocean acoustic tomography: Rays and modes," Rev. Geophys. and Space Phys., vol. 21, pp. 777-793, May 1983.

[14] R. J. Urick, Principles of Underwater Sound, 2nd ed. New York: McGraw-Hill, 1975.

[15] R. C. Spindel, "An underwater acoustic pulse compression system," IEEE Trans. Acoust. Speech, Signal Processing, vol. ASSP-27, pp. 723-728, Dec. 1979.

[16] J. L. Spiesberger, R. C. Spindel, and K. Metzger, "Stability and identification of ocean acoustic multipaths," J. Acoust. Soc. Amer., vol. 67, pp. 2011-2017, June 1980.

[17] T. G. Birdsall, "On understanding the matched filter in the frequency domain," IEEE Trans. Educ., pp. 168-169, Nov. 1976.

[18] K. Metzger, Jr., "Signal processing equipment and techniques for use in measuring ocean acoustic multipath structures," Ph.D. dissertation, Uniy. of Michigan, Ann Arbor, MI, 1983.

[19] Staff, Technical Planning and Development Group, "Serial ASCII Instrumentation Loop for Marine Research Application," in Oceans 81 Conf. Rec., 1981, pp. 296-300.

[20] D. C. Webb, "SOFAR floats for polymode," in Oceans 77 Conf. Rec., 1977, pp. 44B-1-44B-5.

[21] R. C. Spindel, R. P. Porter, and D. C. Webb, "A mobile coherent lowfrequency acoustic range," IEEE $J$. Oceanic Eng., vol. OE-2, pp. 331-337, 1977.

[22] P. F. Worcester, "An example of ocean acoustic multipath identification at long range using both travel time and vertical arrival angle," $J$. Acoust. Soc. Amer., vol. 70, pp. 1743-1747, 1981.

[23] A. Rihaczek, Principles of High-Resolution Radar. New York: McGraw-Hill, 1969.

[24] R. G. Pridham and R. A. Mucci, "Shifted sideband beamformer," IEEE Trans. Acoust., Speech, Signal Processing, vol. ASSP-27, pp. 713-722, Dec. 1979.

[25] G. R. Fox, "Ambient-noise directivity measurements," J. Acoust. Soc. Amer., vol. 36, pp. 1537-1540, Aug. 1964.

[26] E. H. Axelrod, B. A. Schoomer, and W. A. Von Winkle, "Vertical directionality of ambient noise in the deep ocean at a site near Bermuda," J. Acoust. Soc. Amer., vol. 37, pp. 77-83, Jan. 1965.

[27] S. M. Flatté, "Wave propagation through random media: Contributions from ocean acoustics," Proc. IEEE, vol. 71, pp. 1267-1294, Nov. 1983

[28] S. M. Flatté, R. Dashen, W. H. Munk, K, M. Watson, and F. Zachariasen, Sound Transmission Through a Fluctuating Ocean, S. M. Flatté, Ed. London, England: Cambridge University Press, 1979.

[29] T. B. Sanford, "Observations of strong current shears in the deep ocean and some implications on sound rays," J. Acoust. Soc. Amer., vol. 56, pp. 1118-1121, Oct. 1974.

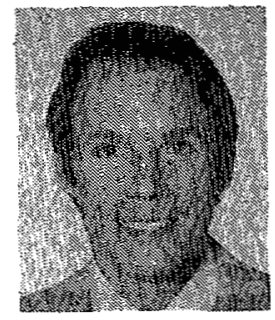

Peter F. Worcester received the B.S. degree in engineering physics from the University of Illinois, Urbana, in 1968, the M.S. degree in physics from Stanford University, Stanford, CA, in 1969, and the $\mathrm{Ph} . \mathrm{D}$. degree in oceanography from the Scripps Institution of Oceanography, University of California, San Diego, La Jolla, in 1977.

He was appointed as Postgraduate Research Oceanographer at Scripps upon receiving his degree, and as Assistant Research Oceanographer in 1978. In 1984 he was appointed to his current 
position as Associate Research Oceanographer at the Institute of Geophysics and Planetary Physics of the Scripps Institution of Oceanography.

Dr. Worcester is a member of Tau Beta Pi and the recipient of Lisle Abbott Rose Memorial Award of the University of Illinois, 1968.

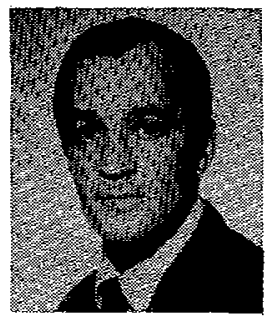

Robert C. Spindel (S'63-M'70) received the Bachelor degree in electrical engineering from the Cooper Union, New York, NY, in 1965 and the M.S. and Ph.D. degrees, also in electrical engineering, from Yale University, New Haven, CT, in 1966 and 1971, respectively.

During 1971 he was a Postdoctoral Research Fellow at Woods Hole Oceanographic Institution, Woods Hole, MA, and in 1972 he joined the Scientific Staff in the Ocean Engineering Department. Currently he is a Senior Scientist and Chairman of the Department of Ocean Engineering at Woods Hole. His primary research interest is underwater acoustics and he has worked on problems in acoustic scattering, propagation, signal processing, navigation, instrument design and development. He is presently engaged in developing instrumentation and techniques to apply ocean acoustic tomography to large-scale ocean measurement. He has authored or coauthored about 50 publications, holds several patents, and has served as Chief Scientist on many research cruises.

In 1981 Dr. Spindel was awarded the A. B. Wood Medal by the British Institute of Acoustics. $\mathrm{He}$ is an Associate Editor of the IEEE JOURNAL OF OCEANIC ENGINEERING and a Fellow of the Acoustical Society of America.

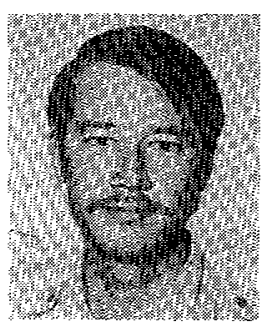

Bruce M. Howe attended Stanford University, Stanford, CA, and received the B.S. and M.S. degrees in mechanical engineering in 1978. While at Stanford he worked on an air-sea interaction project, after which he worked at the University of Karlsruhe on an atmospheric laser Doppler velocimeter.

He is presently at the Scripps Institution of Oceanography, University of California, San Diego, La Jolla, working towards the Ph.D. in Physical Oceanography. 